\title{
Aplicación del Despliegue de la Función de Calidad (QFD) para el rediseño del proceso comercial de una empresa del sector de transporte de mercancía Quality Function Deployment (QFD) methodology application to redesign the commercial process of a merchandise transportation company.
}

\author{
Jasmin Herrera De la Barrera1, Mateo Aparicio Díaz², Jeiston Gutiérrez Pérez², Ingrid Meza Romero², Mery Torres \\ Figueroa $^{2}$
}

\author{
${ }^{1}$ Docente, Fundación Universitaria Tecnológico Comfenalco-Cartagena, Colombia. \\ jherrerad@tecnocomfenalco.edu.co \\ ${ }^{2}$ Estudiante de VI semestre, Tecnología en Control de Calidad, Fundación Universitaria Tecnológico Comfenalco Cartagena, \\ Colombia. mateoapariciodiaz@hotmail.com; jeistongutierrez@gmail.com; ingridmero_1994@hotmail.com;
} merytorrez_18@hotmail.com

Recibido: 15/ago/2019 - Revisado: 30/sep/2019

Aceptado: 30/oct/2019 - Publicado: 30/jun/2019

Resumen Este estudio tiene como objetivo aplicar la metodología Despliegue de la Función de Calidad (QFD) para rediseñar el proceso comercial en una empresa de transporte de mercancías, debido a las fallas y debilidades presentadas en cuanto al manejo, retroalimentación y mantenimiento de los clientes, de manera que se logre mejorar la prestación del servicio, estructurando y agrupando sus actividades en función de la prestación de servicios con altos estándares de calidad y el permanente seguimiento de los mismos. Primeramente, se diagnosticó el proceso comercial a través de entrevistas con el personal encargado, definiendo así etapas del proceso tales como el contacto con el cliente, la presentación de portafolio de servicios, la oferta comercial, la aprobación del cliente, el registro del cliente y por último la prestación del servicio donde se da el momento de la verdad. Posteriormente se caracterizaron los clientes tomando una muestra de 31 clientes fijos de la regional Cartagena, caracterizando y evaluando la competencia potencial de la empresa mediante encuestas y benchmarking. Para la posterior aplicación del Despliegue de la Función de Calidad (QFD), identificando los puntos críticos del proceso, que sirvieron como línea base para el rediseño del proceso comercial, a través de la información obtenida del QFD. A partir de allí se caracteriza y realiza el flujograma del nuevo proceso comercial incorporando las necesidades y expectativas del cliente frente al servicio prestado.

Palabras claves QFD, proceso, caracterización, calidad, cliente.

Abstract This study aims to apply the Quality Function Deployment (QFD) methodology to redesign the business process of a merchandise transportation company, due to the failures and weaknesses found in management, feedback and customer keeping by structuring and grouping the commercial activities in function of service supply with high quality standards and constant monitoring of the process as a way to improve the customer service.At first, the commercial process was diagnosed through interviews to in charge staff determining stages of the process such as customer support, services briefcase presentation, customer endorsement, customer inscription, and the moment of truth: service provision. Later, the clients were characterized by taking a sample of 31 permanent clients from the Cartagena regional; the potential competition of the company was characterized and evaluated through surveys and benchmarking. Then, were identify the process critical points which were used as the basis to apply House of Quality (HOQ) or Quality Function Deployment (QFD) methodology for redesign the commercial process and create a flowchart of the new commercial process that incorporates the needs and expectations of the client in relation to the service provided.

Keywords QFD, process, characterization, quality, customer. 


\section{Introducción}

Es importante que toda empresa satisfaga las crecientes necesidades de sus clientes para mantenerse en el mercado y ser más competitivas respecto a sus iguales; y aún más, en un sector tan versátil como el del transporte.

Actualmente en una empresa de transporte de mercancía y mensajería en la ciudad de Cartagena, existe una desviación en el proceso comercial al no mantener una comunicación asertiva con los clientes fijos de la empresa, presentando fallas y debilidades en cuanto al manejo, retroalimentación y mantenimiento de los mismos, lo cual se evidencia en las PQRS (peticiones, quejas, reclamos y solicitudes), (Intertek, s.f.) de la empresa, donde el análisis de los resultados del último trimestre del año 2018, evidenció que el $86 \%$ de las quejas estaban relacionadas a fallas en la retroalimentación, comunicación, claridad en la información y acompañamiento ofrecido por el área comercial.

En este tipo de negocios se hace sensible la atención, comunicación y respuesta asertiva a los requerimientos realizados, la promesa de valor no solo incluye el despacho de la mercancía en las condiciones pactadas con el cliente, si no en el trato que reciben los mismos, la oportunidad en el servicio, la trazabilidad en la información y la oportunidad en la respuesta, son características esenciales a la hora de ser seleccionados como proveedor. El diseño actual del servicio ofrecido por la empresa objeto de estudio, está orientado a la entrega y distribución de la mercancía, dejando de lado la calidad del servicio como tal.

Este esquema de operación y las evidencias que describen la insatisfacción del cliente, sugiere la adopción de una metodología que articule las necesidades del cliente con la capacidad de la organización, definiendo como la más adecuada, la casa de la calidad o el despliegue de la función de calidad (QFD, por sus siglas inglesas), la cual es un método de diseño aplicado a productos, procesos y servicios que recoge las necesidades y expectativas de los clientes y las traduce en pasos sucesivos, a características técnicas y operativas satisfactorias. (Delgado, Parra, \& Suarez, 2015).

El objetivo de este trabajo fue rediseñar el proceso comercial de la empresa de transporte de mercancía y mensajería, de manera que se estructuren y agrupen sus actividades en función de la prestación de servicios con altos estándares de calidad y el permanente seguimiento de los mismos, fortaleciendo las relaciones con sus clientes; a partir de allí, se estructuran tres etapas constitutivas del QFD: fase1 caracterización del proceso, es decir la definición del cómo, fase 2 identificación de las necesidades y expectativas del cliente, definición de los qué, y la última fase evaluación del proceso a partir de las buenas prácticas de la competencia.

\section{Antecedentes}

Los orígenes de la metodología QFD se remontan a finales de los años 60 en Tokio, cuando el profesor Yoki Akao, de la Universidad de Tamagawa, empezó a expresar las necesidades de los clientes en puntos críticos para el aseguramiento de la calidad, y planteó que se tuvieran en cuenta a través de las etapas de diseño, hasta la fabricación de los productos. Pero fue en 1972, con la ayuda de los doctores Mizuno y Furukawa, en el astillero de Kobe que se desarrolló la matriz de demandas del cliente y características de calidad, formalizando el concepto de Akao y dando como fruto el desarrollo de la metodología QFD (Olaya, Cortés, \& Duarte, 2005).

La metodología QFD es un método de diseños de productos y servicios que recoge la voz del cliente y la traduce en pasos sucesivos, a características de diseño y operación que satisfacen las demandas y expectativas del mercado (Yacuzzi \& Martín, 2013). Se puede definir QFD como el despliegue, paso a paso, con el mayor detalle, de las funciones u operaciones que conforman sistemáticamente la calidad, convirtiendo las demandas de los consumidores en características concretas de calidad, para proceder a desarrollar una calidad de diseño mediante el 
despliegue sistemático de relaciones entre demandas y características, comenzando por la calidad de cada componente funcional y extendiendo el despliegue a cada parte o proceso, apoyándose en la principal herramienta para conseguir estos fines; el grafico denominado como "la casa de la calidad" (Miranda, Chamorro, \& Rubio, 2007).

El despliegue de la función de calidad sirve como norma para la evaluación del grado de cumplimiento de las expectativas y necesidades del cliente, teniendo como objetivos básicos, primero hacer llegar claramente a todos los empleados la voz del cliente y segundo, satisfacer, en primer lugar las necesidades del cliente.(York, 1994).

Como lo demuestran desde el 2014 investigaciones realizadas en Centroamérica, donde se emplea el QFD en el diseño de una montura mecánica para el desarrollo de un producto innovador que garantiza la satisfacción del cliente (Montesinos, Echeverría, Alvarado, \& Manzano, 2016). Igualmente en Suramérica, en Colombia, en el año 2011, se desarrolló un aplicativo de acuerdo con las especificaciones obtenidas al utilizar la metodología del despliegue de la función de la calidad (QFD) en un grupo de población rural del municipio de Zipacón (Cundinamarca) para la producción y comercialización de papa (Vélez, 2011). Y en el departamento de Bolívar, en la ciudad de Cartagena, en el año 2017, se utilizó el despliegue de la función de la calidad para establecer la propuesta de diseño de una guía metodológica para la implementación de sistemas de gestión en el sector de la construcción, replicable en cualquier otro ramo de la economía colombiana.(Herrera De la Barrera \& Narváez, 2017).

En 2017 en Europa se implementó la función de calidad (QFD) en el sector turístico, con el fin de determinar la calidad del proceso a partir de las demandas de los clientes (Kurtulmuşoğlu \& Pakdil, 2017). En Asia, en el año 2018, a través de la metodología QFD se diseñó un nuevo sistema en el PT. Global Green Trading determinando los atributos y requerimientos de los consumidores (Imaroh, 2018). Asimismo, en México la metodología QFD fue aplicada en el sector de la educación para el diseño de una propuesta que ilustra la evaluación de calidad de cuatro unidades del Sistema de Bibliotecas de la Universidad de Guadalajara, determinando los aspectos a mejorar en el proceso y los niveles de calidad con los que cuenta la Institución Educativa (Gutierrez, Gutierrez, Garibay, \& Díaz, 2014). De igual manera, en Atlántico, departamento de Colombia, en el 2004, se aplicó la metodología QFD en una empresa del sector de confecciones de la ciudad de Barranquilla, en el diseño de una metodología de mejora de procesos, para brindar una herramienta de mejoramiento continuo, sencilla, completa y de fácil implementación para directores de pequeñas $y$ medianas empresas, que les permite obtener resultados positivos en todas las áreas(Nuñez, Vélez, \& Berdugo, 2004). Se puede afirmar, a partir del anterior rastreo literario, que la metodología QFD ha sido aplicada con éxito en el rediseño de procesos de prestación de servicios, con el objeto de mejorar la percepción que el cliente tiene frente a la prestación del mismo.

\section{Metodología}

\subsection{Diseño}

La presente Investigación es de tipo proyectiva (Barrera, 2000), que busca elaborar el rediseño del proceso comercial de la empresa objeto de estudio, a través de la aplicación de la metodología de la casa de la calidad (QFD), diagnosticando el proceso, caracterizando sus clientes y competidores.

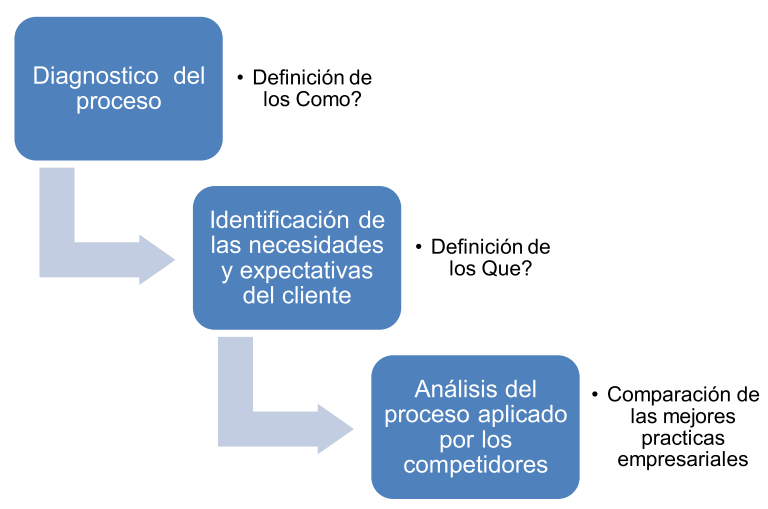

Ilustración 1 Descripción metodológica 


\subsection{Procedimiento}

La estructura metodológica desarrollada es orientada de acuerdo al modelo del QFD, iniciando en la primera fase con el diagnóstico del proceso comercial de la empresa, utilizando como técnica visitas de campo y como herramienta de recolección de datos, una guía de observación orientada hacia la prestación del servicio, definiendo las características técnicas (los “¿Cómo?”), las dificultades o fallas que se pueden presentar durante el desarrollo del mismo, logrando así caracterizar el proceso determinando cada una de las etapas orientadas a demostrar que se hace y como se hace.

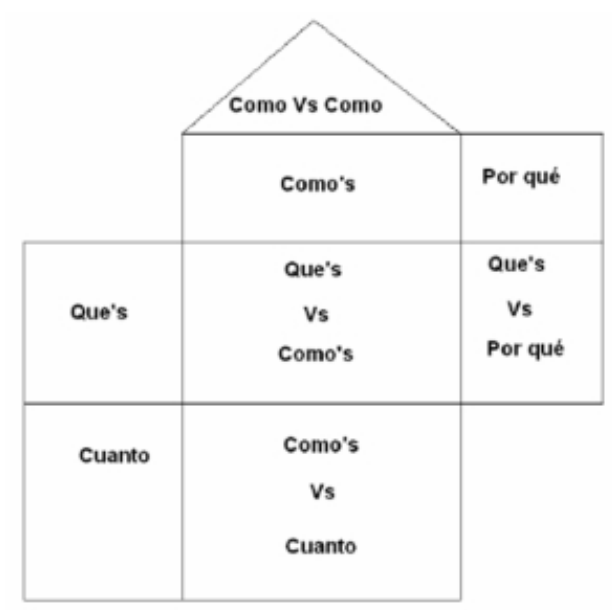

Ilustración 2 Metodología del despliegue de la función de la calidad. Fuente: (UDLAP, 2017)

De igual forma se caracteriza a los clientes, a través de la aplicación de encuestas a una muestra de 35 clientes fijos de la Regional Cartagena lo que definirá los ¿qué? de los que habla la metodología. (Requerimientos, necesidades y expectativas).

En la tercera fase se caracterizará la competencia mediante la aplicación de una encuesta orientada a recolectar información pertinente sobre cómo desarrolla la competencia, los procesos de prestación del servicio.

En la cuarta fase se aplica la matriz de interrelaciones para evaluar que tanto las características técnicas satisfacen los requerimientos del cliente, de igual forma se calculan las características de control para obtener las ponderaciones absolutas y relativas de las características técnicas.

Finalmente se realizará el despliegue de la función de la calidad, determinado los puntos críticos y áreas de mejora procediendo a realizar la caracterización del nuevo proceso con las mejoras derivadas del diseño del proceso.

\subsection{Análisis de datos}

Para obtener la muestra de los clientes fijos, se trabajó con la base de datos de los clientes que tienen cuenta fija con la regional de Cartagena, la cual cuenta con una población total de 71 clientes. Para el cálculo de la muestra se utilizó la fórmula de muestreo aleatorio simple para población finita para una proporción.(Aguilar-Barojas, 2005)

En donde:

$$
n=\frac{N * Z_{\alpha / 2}{ }^{2} * p * q}{d^{2} *(\mathrm{~N}-1)+Z_{\alpha / 2}{ }^{2} * p * q}
$$

$\mathrm{N}=$ Tamaño de la población

\begin{tabular}{ccc}
\hline Población & $\mathrm{N}$ & 71 \\
Nivel Confianza & $1-\alpha$ & 0,90 \\
Nivel Significancia & $\alpha$ & 0,10 \\
& $\alpha / 2$ & 0,05 \\
Valor normalizado. & $\mathrm{Z} \alpha / 2$ & 1,645 \\
Prob. Éxito & $\mathrm{p}$ & 0,5 \\
Prob. Fracaso & $\mathrm{q}$ & 0,5 \\
Precisión & $\mathrm{d}$ & 0,1 \\
\hline
\end{tabular}

Dando como resultado una muestra de 35 clientes.

\section{Resultados y Discusión}

\subsection{Diagnostico del proceso}

El diagnóstico realizado a la calidad de la prestación del servicio (Buonocore, 1980), determinó las actividades puntuales desarrolladas durante el 
proceso y las dificultades que se presentan, esta información es organizada en un diagrama de bloques, ver figura 1 , donde se representa gráficamente la secuencia de las actividades y otros eventos que ocurren en el proceso (Gutierrez H. D., 2013), utilizando la simbología estandarizada por el Instituto Nacional Estadounidense de Estandarización (American Nacional Institute ANSI) para la diagramación de diagramas de bloques. (Medina, 2005):

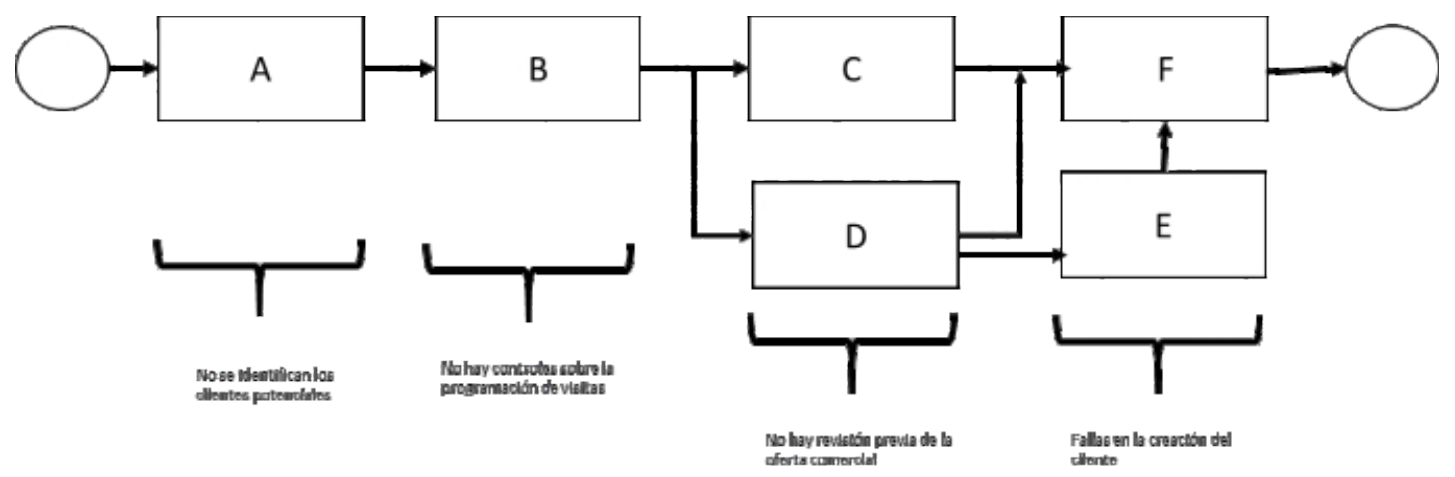

Fig. 1. Diagrama de bloques del proceso comercial de la empresa

A: Contacto con el cliente

B: Presentación del portafolio de servicios

C: Oferta comercial

El diagnostico permitió tener una visión más clara del desarrollo de cada una de las etapas del proceso, de las actividades que hacen falta e identificar la problemática que sobreviene de cada actividad del proceso comercial y su impacto en el mismo, determinado 6 actividades claves (Contacto con el cliente, presentación del portafolio de servicios, oferta comercial, aprobación del cliente, registro del cliente en el sistema de la empresa, prestación del servicio) y 4 fallas principales identificadas como causa de la insatisfacción (no identificación de los clientes potenciales, falta de controles en la programación de visitas, revisión previa de las ofertas comerciales, falla en la creación de clientes). Estas fallas representan el $80 \%$ de las causas de los problemas en cada una de las etapas identificadas.

\subsection{Caracterización del cliente}

A partir de la información obtenida del proceso comercial, se procedió a caracterizar a los clientes, donde se obtienen los requerimientos, síntesis de los principales componentes de la voz del cliente. (Yacuzzi
D: Aprobación del cliente

E: Registro del cliente en el sistema de la empresa

F: Prestación del servicio

\& Martín, 2013); para ello, durante los meses de Abril y Mayo del 2019, se realizó, a una muestra de 36 clientes fidelizados con la empresa, una encuesta, que permitió recolectar información pertinente de las necesidades y expectativas de estos.

Las respuestas obtenidas por los clientes se encuentran distribuidas equitativamente dentro de los intervalos de las edades garantizando un juicio imparcial con respecto a las respuestas proporcionadas en las encuestas, distribución observada en el grafico 1.

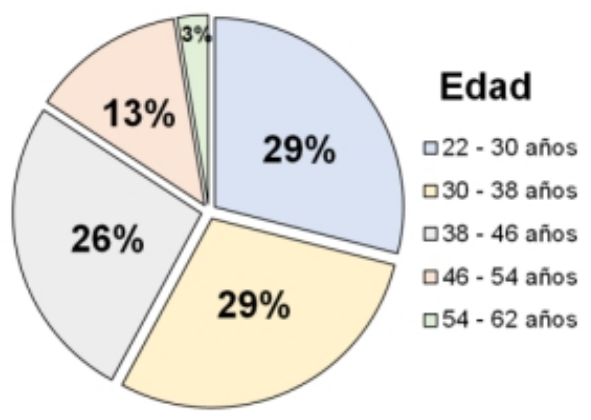

Gráfico 1. Distribución etaria. 
La grafica 2 representa la perspectiva que tienen los clientes al momento de clasificar las características de respuesta oportuna, acompañamiento por parte del asesor, comunicación asertiva, claridad en los acuerdos comerciales y calidad del servicio se evidencia en una clasificación según la importancia definida por los clientes encuestados.

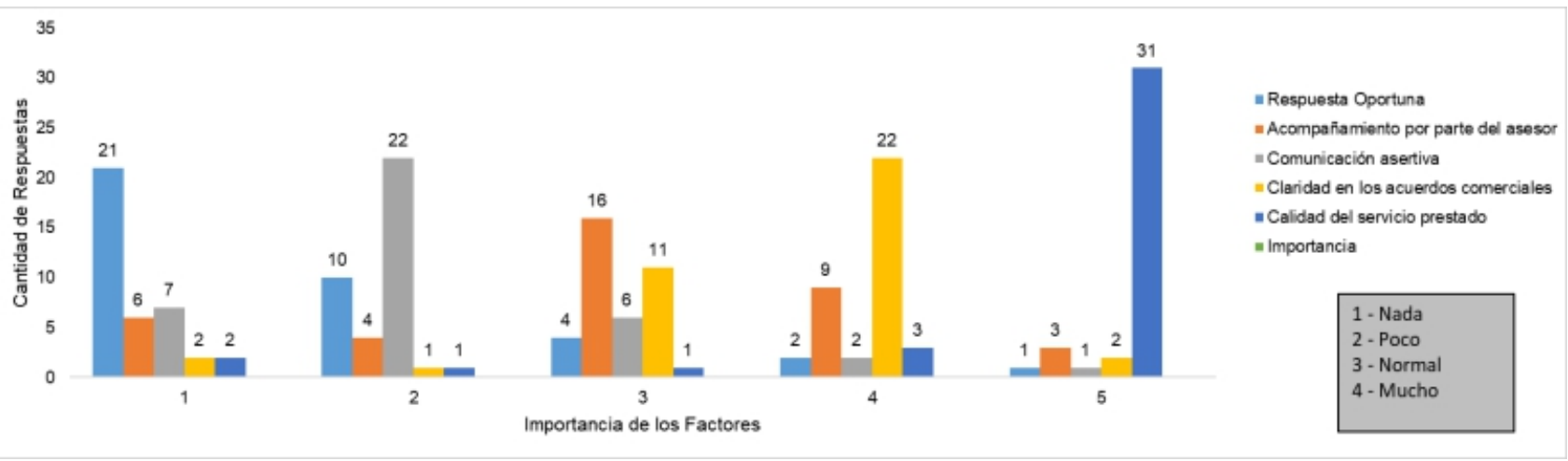

Gráfico 2. Perspectiva de los clientes.

El $81,57 \%$ de la muestra encuestada le otorga demasiada importancia y prioridad a la calidad del servicio, siendo este un factor clave al momento de recibir una buena atención y en el cual se integran las demás características evaluadas, el 55,26\% afirmaron una importancia nula con respecto a las respuestas oportunas.
Una vez recopilada toda la información obtenida de la muestra de las encuestas, se organizó y tabuló de acuerdo a la tabla 1, cada uno de los requerimientos y expectativas manifestadas por los clientes frente al servicio.

Tabla 1. Requerimientos y expectativas manifestadas por los clientes en la encuesta

\begin{tabular}{|c|c|c|c|c|}
\hline ID & Requerimientos & Frecuencia & $\mathbf{\%}$ & $\mathbf{\%}$ Acum \\
\hline CAR 1 & Canal de Comunicación & 45 & $21,63 \%$ & $21,63 \%$ \\
\hline CAR2 & Respuesta Oportuna & 25 & $12,02 \%$ & $33,65 \%$ \\
\hline CAR 3 & Innovación Tecnológica & 22 & $10,58 \%$ & $44,23 \%$ \\
\hline CAR4 & Acompañamiento comercial & 21 & $10,10 \%$ & $54,33 \%$ \\
\hline CAR5 & Cambios en los canales de comunicación & 19 & $9,13 \%$ & $63,46 \%$ \\
\hline CAR6 & Más interés por los clientes & 13 & $6,25 \%$ & $69,71 \%$ \\
\hline CAR7 & Tarifas especiales (Promociones) & 12 & $5,77 \%$ & $75,48 \%$ \\
\hline CAR8 & Calidad de Servicio al cliente & 11 & $5,29 \%$ & $80,77 \%$ \\
\hline CAR9 & Que el área comercial visite las empresas & 10 & $4,81 \%$ & $85,58 \%$ \\
\hline CAR10 & Tiempos de Respuesta & 8 & $3,85 \%$ & $89,42 \%$ \\
\hline CAR11 & Otras & 7 & $3,37 \%$ & $92,79 \%$ \\
\hline CAR12 & Relaciones Comerciales & 6 & $2,88 \%$ & $95,67 \%$ \\
\hline CAR13 & Mejorar enfoque a cliente & 6 & $2,88 \%$ & $98,56 \%$ \\
\hline CAR14 & Hacer seguimiento de asesores & 3 & $1,44 \%$ & $100 \%$ \\
\hline & Total & 208 & $100 \%$ & \\
\hline
\end{tabular}

De esta manera se obtienen las diversas perspectivas que tienen los clientes de acuerdo al servicio prestado por la empresa. Para priorizar las posibles variables que generan un mayor grado de satisfacción para los clientes se hizo necesario utilizar el diagrama de Pareto, el cual es una herramienta 
estadística que permite priorizar las variables que por lo general producen la mayor parte de las problemáticas en las empresas y así poder atacarlas en mayor medida, de ahí su particular nombre del diagrama de (80-20) grafico 3 , donde el $80 \%$ de los problemas se encuentran inmersos en el $20 \%$ de las causas, de esta forma se pueden diferenciar los muchos triviales de los pocos vitales, los cuales son los que se utilizarán para aumentar el nivel de satisfacción de los clientes al momento de recibir el servicio comercial.(Lopéz, 2016)

Una vez definidas las características deseadas por el cliente se procede a realizar el grafico de Pareto

En el grafico 3 se puede observar que las expectativas presentadas por el cliente están distribuidas en grupos de intereses similares, de tal manera que deben ser agrupadas para una mejor priorización y asignación de importancia para las necesidades y requerimientos que posee el cliente al momento de prestar el servicio comercial. Para el agrupamiento de las características de valor se utilizó un diagrama de afinidad (Figura3) el cual permite agrupar atributos que tienen similar naturaleza. El diagrama de afinidad es en sí una técnica que ayuda a organizar grandes cantidades de datos obtenidos, ya sea de una lluvia de ideas, de una encuesta o alguna otra técnica de recolección de datos, agrupando así, elementos que están relacionados entre sí de manera natural, para entender la estructura de un problema global mediante el análisis de las afinidades verbales(Madrigal, 2018). De esta forma se pretende dar más entendimiento y ser más representativos al momento de definir las nuevas características de calidad del proceso comercial y a su vez contenga todos los posibles requerimientos y expectativas de los clientes que fueron capturados mediante la encuesta. Estos grupos de características definen verdaderamente lo que el cliente está necesitando al momento de requerir los servicios comerciales de la empresa

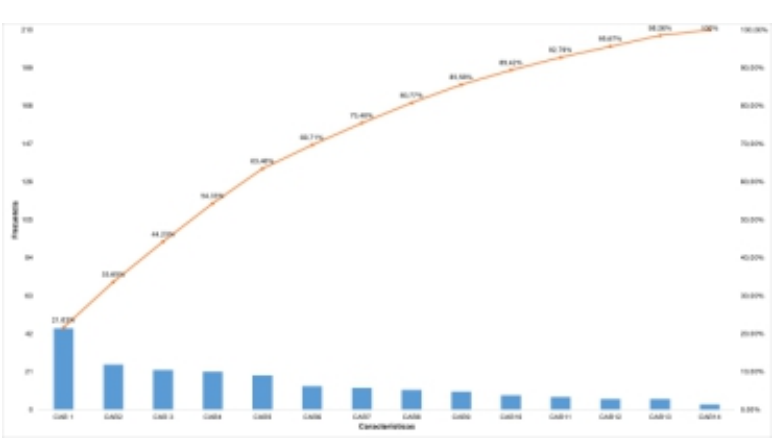

Gráfico 3. Diagrama de Pareto "Requerimientos y expectativas de los clientes

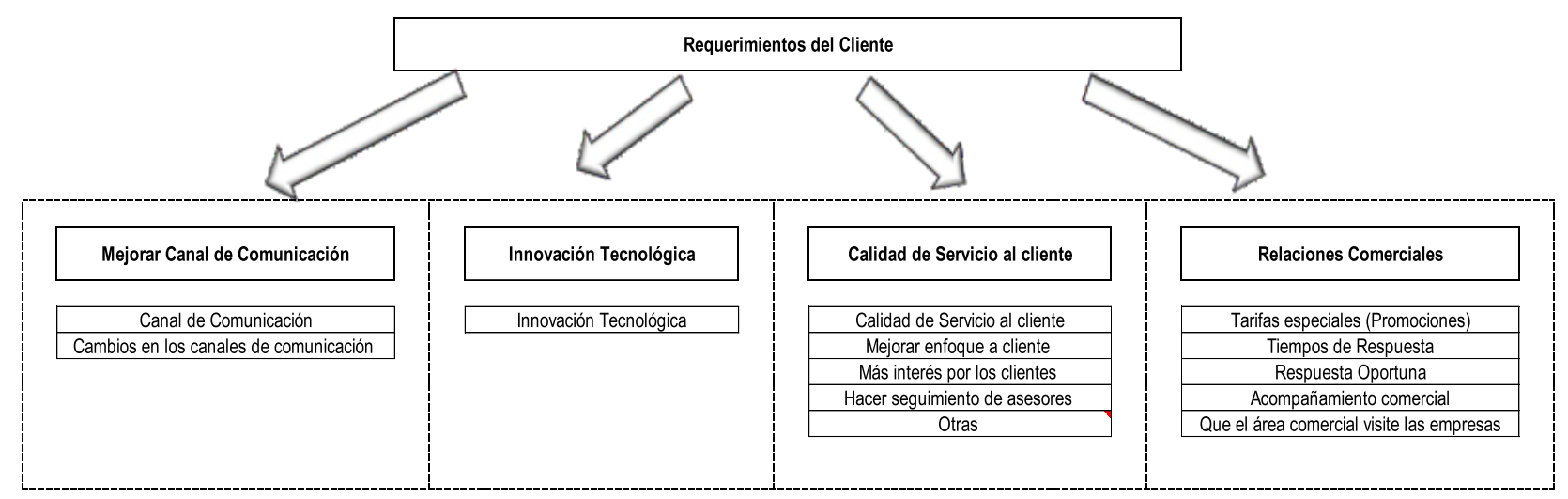

Fig. 3. Diagrama de afinidad.

Una vez identificadas las características globales se procede a realizar un diagrama de Pareto de segundo nivel gráfica 4 , que permite priorizar y asignar un grado de importancia a cada una de ellas, la priorización se basó en la opinión recolectada mediante las encuestas y así mismo la cantidad de 
opiniones que encajan dentro del grupo que se está tomando como referencia, de esta manera se tuvieron en cuenta la totalidad de respuestas emitidas en la asignación del grado de importancia del cliente de acuerdo a las características definidas por el mismo.

La gráfica 4 representa el grado de importancia de las características requeridas por los clientes definiendo así los denominados ¿qué? (los requerimientos que hay que tener en cuenta en el diseño dado que son las necesidades y expectativas del cliente.

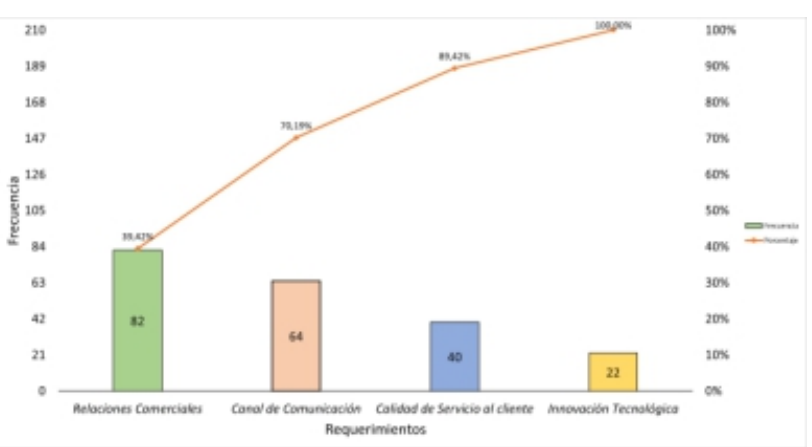

Gráfico 4. Diagrama de Pareto de segundo nivel.

En el gráfico 4 se puede apreciar que el $39,42 \%$ de los clientes prefiere un mejor manejo de las relaciones comerciales y luego un adecuado canal de comunicación que permita la correcta y fluida trasmisión de la información que es de importancia para los clientes, la calidad del servicio no le es indiferente, pero dentro del grupo de expectativas también juega un papel importante, así como las innovaciones tecnológicas en el servicio prestado

\subsection{Caracterización de la competencia}

Para la caracterización de la competencia de la empresa, se aplicó una encuesta a 3 de las principales empresas competidoras del sector de transporte, logrando captar las características y el nivel del servicio que estas brindan a sus clientes, mediante un análisis de Benchmarking competitivo, el cual consiste básicamente en un intercambio know how entre empresas, considerado como una pieza clave en los programas de reingeniería y aplicado en el QFD como una herramienta para la comparación del producto o servicio de la empresa con el de la competencia (Llontop, 2011), se caracterizó y comparó la calidad del servicio prestado acompañado por una escala de Likert la cual nos permite definir mediante niveles la actitud de las personas hacia ideas, objetos o situaciones y el grado de acuerdo o desacuerdo respecto a cada una de ellas (Plazas, 2011). Por lo tanto, se precisó el grado de cumplimiento que estos le otorgan a las expectativas, necesidades y requerimientos que se están evaluando del proceso comercial y conociendo el grado en que satisfacen los requerimientos de los clientes.

El grafico 5 indica el grado en que cada empresa del sector cumple con los requerimientos establecidos por el cliente, concluyendo que la empresa objeto de estudio no aplica a cabalidad los requisitos del cliente, mientras que las empresas del sector han dado respuesta a algunos de esos requerimientos, aunque no en un alto grado de implementación.

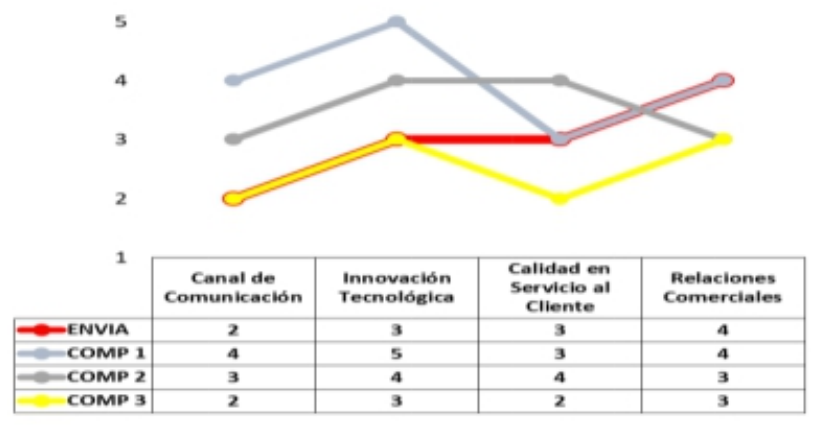

Gráfico 5. Evaluación competitiva.

Las características técnicas o los ¿Cómo? que permitieron dar respuesta a las expectativas de los clientes, son cuantificadas y representan una repuesta global del cliente, debido a que se trata de parámetros que deben poder controlarse y medir para satisfacer las necesidades identificadas, al igual que las necesidades del cliente las características de calidad deben reorganizarse y agruparse(Ministerio de la Protección Social, 2019). Para ello se realizó una lluvia de ideas de las posibles herramientas a utilizar para satisfacer los requerimientos de los clientes. Debido a que los "cómo" juegan un papel fundamental al 
Herrera de la Barrera, J., Aparicio Díaz, M., Gutiérrez Pérez, J., Meza Romero, I., \& Torres Figueroa, M. (2019). Aplicación del Despliegue de la Función de Calidad (QFD) para el rediseño del proceso comercial de una empresa del sector de transporte de mercancía. Teknos Revista Científica, 19(2), 61-74.

momento del rediseño del proceso como tal, se realizó una encuesta interna con el personal que brinda el servicio para que ellos escogieran a juicio personal, aplicando la experiencia obtenida, las mejores características técnicas, los resultados se muestran en la tabla 2 En esta tabla se pueden observar los "Cómo" que serán contemplados en el proceso de rediseño y a su vez el beneficio que traen con respecto a los requerimientos evaluados.

Tabla 2. Características técnicas seleccionadas.

\begin{tabular}{|c|c|c|c|c|c|c|c|}
\hline \multirow{5}{*}{ ¿Qué? } & & \multicolumn{6}{|c|}{ ¿Cómo? } \\
\hline & Mejorar Canal de Comunicación & Chat Online intemo & $\begin{array}{c}\text { (Canal de comunicación Comercial- } \\
\text { Operaciones para mejojar coordinación en } \\
\text { cuanto la disponibilidad de horarios de } \\
\text { recolección) }\end{array}$ & Capacitación en el manejo de las TIC & $\begin{array}{c}\text { (Capacitar a los asesores en el manejo } \\
\text { de las TIC's para el apoyo y } \\
\text { automatización de todas las actividades } \\
\text { del proceso comercial) }\end{array}$ & Chat Online Clientes & $\begin{array}{c}\text { (Brindar un servicio de mejor calidad } \\
\text { y comunicación mas fluida) }\end{array}$ \\
\hline & Innovación Tecnológica & Plataforma Virtual de Servicio & $\begin{array}{c}\text { (Canal de comunicación Cliente-Asesor para } \\
\text { garantizar una comunicación asertiva al } \\
\text { cliente y retroalimentación al servicio) }\end{array}$ & Redes sociales & $\begin{array}{c}\text { (Establecer canales de comunicaciones } \\
\text { donde los clientes puedan interactuar } \\
\text { libremente, conocer, comentar y opinar } \\
\text { con respecto a los servicios brindados } \\
\text { por la empresa) }\end{array}$ & & \\
\hline & Calidad de Servicio al cliente & $\begin{array}{c}\text { Monitoreo periódico de Satisfacción } \\
\text { del cliente }\end{array}$ & $\begin{array}{c}\text { (Encuesta a los clientes sobre la percepción } \\
\text { del servicio y cumplimento a los } \\
\text { requerimientos) y (Verificación de satisfacción } \\
\text { del cliente mediante llamadas telefonicas) }\end{array}$ & $\begin{array}{l}\text { Reentrenamiento en atención y } \\
\text { mantenimiento de clientes }\end{array}$ & $\begin{array}{l}\text { (Capacitaciones a los asesores que } \\
\text { presenten bajo desempeño en el } \\
\text { servicio prestado) }\end{array}$ & $\begin{array}{l}\text { Indicadores de seguimiento a las } \\
\text { visitas realizadas a los clientes }\end{array}$ & $\begin{array}{l}\text { (Garantizar el control y seguimiento } \\
\text { de los clientes mediante indicadores } \\
\text { de cumplimiento a los asesores que } \\
\text { prestan el servicio) }\end{array}$ \\
\hline & Relaciones Comerciales & Gestión del Cambio & $\begin{array}{l}\text { Anuncios periódicos de los cambios } \\
\text { comerciales y tarifas a los clientes fijos. }\end{array}$ & Programación de visitas comerciales & $\begin{array}{l}\text { (Realizar una programación donde se } \\
\text { realicen visitas a los clientes que se } \\
\text { asocien a sus requerimientos y } \\
\text { solicitudes) }\end{array}$ & Incentivos a clientes potenciales & $\begin{array}{l}\text { (Premiar con tarifas especiales y } \\
\text { descuentos a los clientes que } \\
\text { mensual presenten una alta } \\
\text { utilización del servicio) }\end{array}$ \\
\hline
\end{tabular}

Continuando con el proceso de rediseño, se realiza la matriz de interrelaciones, para identificar cuáles son las relaciones entre los "Qué" y los "Cómo", es decir, donde se compara la voz del cliente con cada una de las características técnicas, dándole una ponderación establecida, ya sea desde un análisis cualitativo o mediante una revisión de las evidencias disponibles. Se utilizan una serie de valores o iconos que indican la mayor o menor la relación: (9) Relación fuerte, (3) Relación media y (1) Relación débil. (Ruiz, 2011)

Tabla 3. Ponderación para la comparación de la voz del cliente y las características técnicas.

Grado de Correlación Símbolo Utilizado Valor Numérico Asignado

Muy correlacionados $\quad$ - 9

Correlacionados $\quad 0 \quad 3$

Poco Correlacionados $\quad \Delta \quad 1$

Sin Relación Blanco $\quad 0$

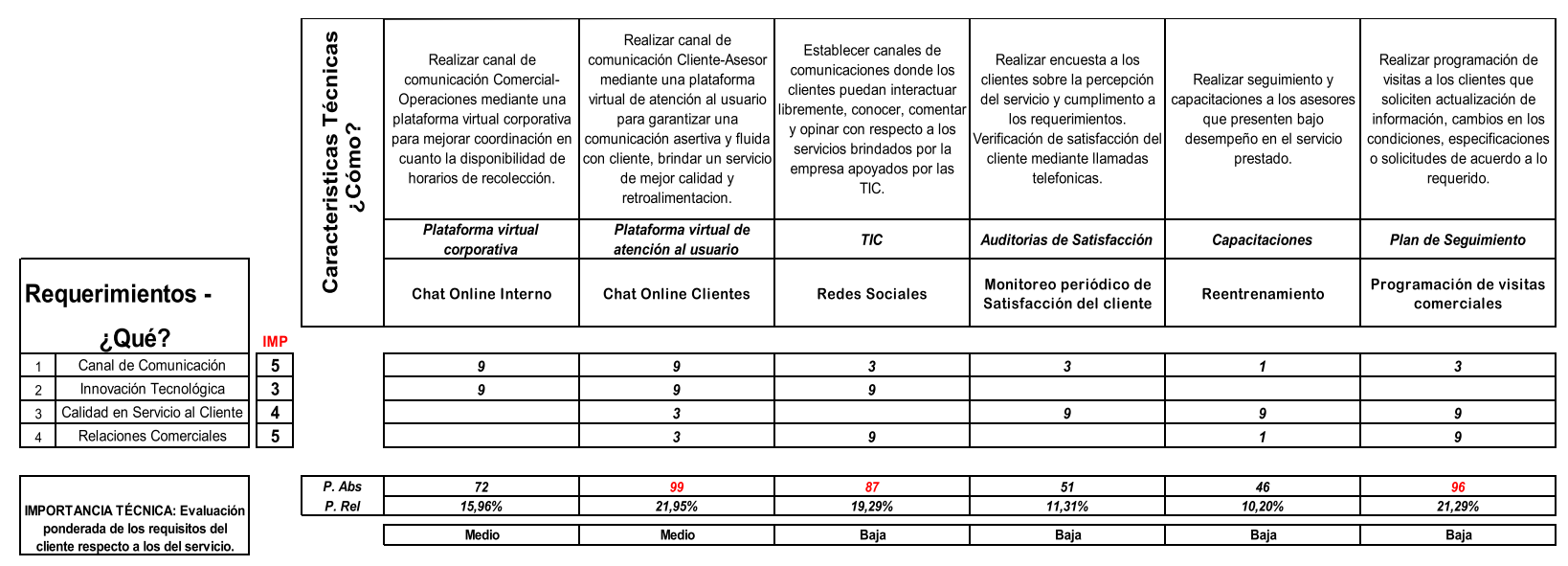

Fig. 5. Matriz de interrelaciones. 
De acuerdo a los requerimientos establecidos por los clientes y las características técnicas que fueron sometidas a selección por parte del personal interno de la empresa, se califica la interrelación que tiene cada uno de los Que y los Cómo, de esta manera se obtiene la matriz de interrelaciones, figura 5, cabe mencionar que donde la interrelación es nula se debe a que ese componente o característica no beneficia o ayuda al cumplimiento del requerimiento establecido por el cliente. Una vez calificada la matriz de interrelaciones se calculan las ponderaciones absolutas y relativas mediante la multiplicación y suma de cada uno de los grados de interrelación de las características y los requerimientos con su respectiva importancia(Zaïdi, 1993), de esta forma se sumaron cada factor de los requerimientos y cada una de las características técnicas; es decir que cada valor numérico asignado para cada cómo y la importancia que tiene para el cliente dicho requerimiento, de esta forma se obtiene la ponderación absoluta, figura 6 , para luego calcular la ponderación relativa que es la proporción de la ponderación absoluta comparado con el total, de esta manera se obtuvieron las condiciones críticas del proceso estableciendo lo que realmente desea el cliente del servicio prestado.(Yacuzzi \& Martín, 2013)

\begin{tabular}{|c|c|c|c|c|c|c|c|}
\hline P. Abs & 72 & 99 & 87 & 51 & 46 & 96 & 451 Total \\
\hline P. Rel & $15,96 \%$ & $21,95 \%$ & $19,29 \%$ & $11,31 \%$ & $10,20 \%$ & $21,29 \%$ & \\
\hline & Medio & Medio & Baja & Baja & Baja & Baja & Dificultad \\
\hline
\end{tabular}

Fig.6. Ponderaciones absolutas y relativas.

\subsection{Calculo de la importancia técnica}

La importancia técnica es la evaluación de la ponderación de los requisitos del cliente con respecto a los servicios recibidos.(Bolar, Tesfamariam, \& Sadiq, 2017), este grado de importancia se clasifica en extremadamente más importante, (alto) representado por 90 o 100, muy importante medio representado por un 70 , fuertemente menor a 69 , moderadamente más importante representado por 30 e igualmente importante representado por un 10 (International Standard Organitation ISO, 2015)

A su vez, se estableció el grado de dificultad que tienen la materialización de las características técnicas frente al servicio, donde las características que tiene una mayor ponderación son alcanzables y la dificultad para conseguirlas es baja. Se realiza la evaluación comparativa de las características técnicas, demostrada en el grafico 6, donde se comparó cuantitativamente el servicio ofrecido por la empresa con respecto a los principales competidores teniendo en cuenta las características técnicas asociadas donde el nivel de la empresa objeto de estudio con respecto a la competencia no cumple con los estándares, lo cual indica que tiene que mejorar en los requerimientos fortaleciendo las herramientas para satisfacer a los clientes.

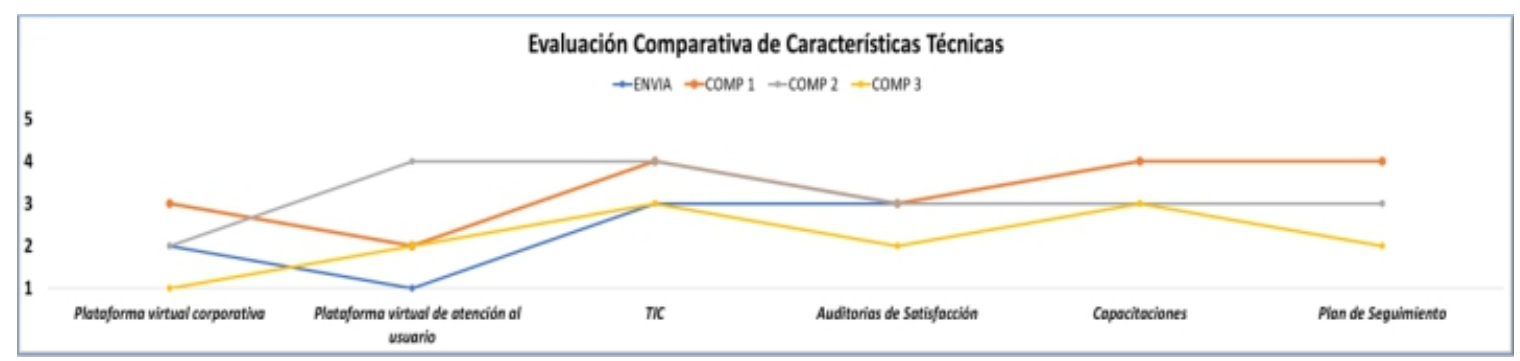

Gráfico 6. Evaluación comparativa de características técnicas 


\subsection{Calculo de la correlación entre variables técnicas}

La matriz de correlación técnica se utiliza para identificar las posibles relaciones entre los "Cómo". Esta es una gráfica triangular, figura 7 , que configura el tejado de la casa de la calidad, que relaciona todos los "cómos" entre sí, estableciendo la posible correlación existente entre ellos. Se utiliza una simbología determinada que puede ser: (X) Correlación Negativa, (O) Correlación Positiva y (•) Correlación Fuertemente Positiva. (Cuatrecasas, 2012)

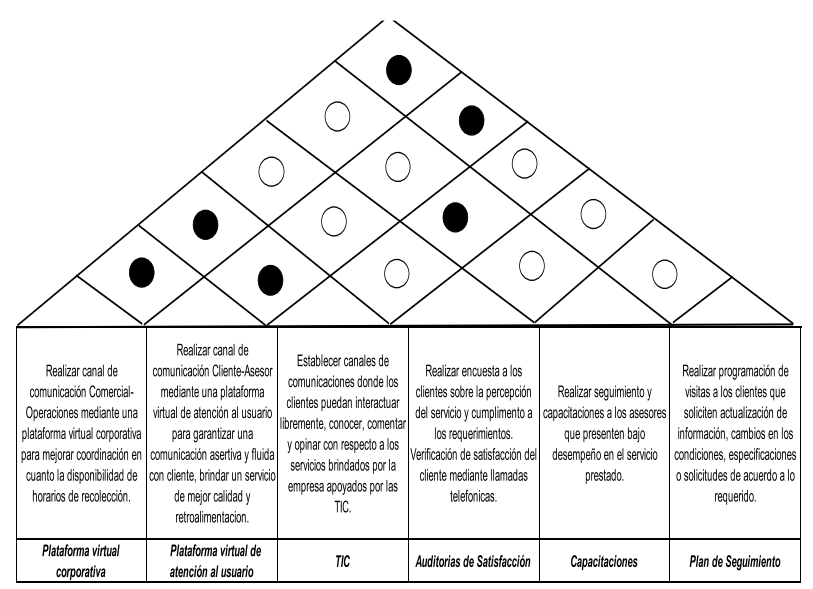

Fig. 7. Matriz de correlación técnica.

Como se puede apreciar en la figura 7 , no existe correlaciones negativas entre las características técnicas, ni mucho menos que sean excluyentes de tal manera que la presencia de uno, no implica afectación en el funcionamiento de la otra. (Montgomery, 2013), se evidencian dos puntos en los cuales se presenta una correlación positiva como son en las plataformas virtuales, ya que estas van de la mano y permiten aumentar la comunicación asertiva entre los clientes externos e internos.

Una vez realizadas todas las etapas del QFD se obtiene el despliegue la función calidad con cada uno de sus componentes que permiten establecer los aspectos críticos y áreas de mejora para que el proceso satisfaga mayormente las necesidades $y$ requerimientos presentados por el cliente.

\subsection{Diseño del proceso comercial}

El despliegue de la función de la calidad permitió el rediseño del proceso comercial. Realizando la caracterización del nuevo proceso, la cual se representa en un diagrama de tortuga, figura 9 , donde se muestra a través de la simulación del cuerpo de una tortuga, utilizando las cuatro patas para representar cuatro preguntas sobre un proceso (Con quién, con qué, cómo, cuántos) y la cabeza y la cola para representar las preguntas sobre los insumos del proceso (qué debemos recibir) y los productos del proceso (qué debemos entregar para satisfacer las expectativas, y en el medio de todo, donde iría el caparazón de la tortuga, se coloca el nombre del proceso. (Gehisy, 2017)

En la figura 9 se observa cómo se han articulado las características definidas por el cliente dentro de los cómo, generando de igual forma indicadores de desempeño que permitirán mantener el proceso dentro de los parámetros de control y monitorear el normal desempeño del mismo; la identificación de recursos y responsables hace que la comprensión del proceso y puesta se realice de forma ordenada y armónica, de igual forma la identificación de la interacción de este proceso con los otros procesos de la organización con la definición de las entradas y salidas, permite ver los productos y los estándares, quien recibe la información y las características con las que es entregado al siguiente proceso

\section{Conclusiones y Recomendaciones}

El proceso comercial de la empresa objeto de estudio presentaba problemáticas en la atención y comunicación con los clientes, variables que son críticas al momento de fidelizar al cliente.

Los clientes presentaron expectativas y requerimientos que inicialmente no se le realizaban seguimientos y control del proceso, de tal manera se generaba insatisfacción de los mismos.. 


\section{teknes}

Herrera de la Barrera, J., Aparicio Díaz, M., Gutiérrez Pérez, J., Meza Romero, I., \& Torres Figueroa, M. (2019). Aplicación del Despliegue de la Función de Calidad (QFD) para el rediseño del proceso comercial de una empresa del sector de transporte de mercancía. Teknos Revista Científica, 19(2), 61-74.

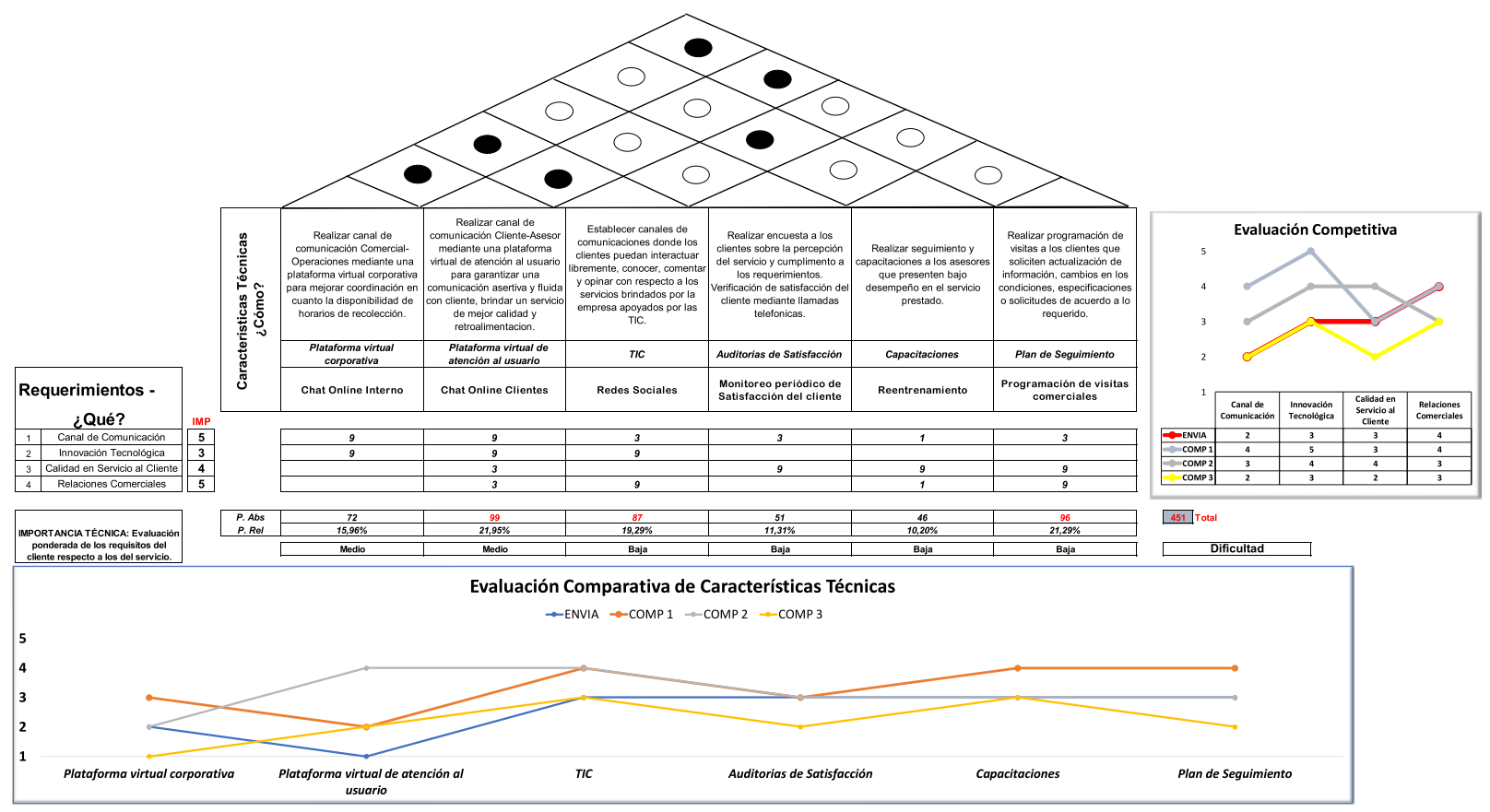

Fig.8. Despliegue de la función de la calidad o casa de la calidad.

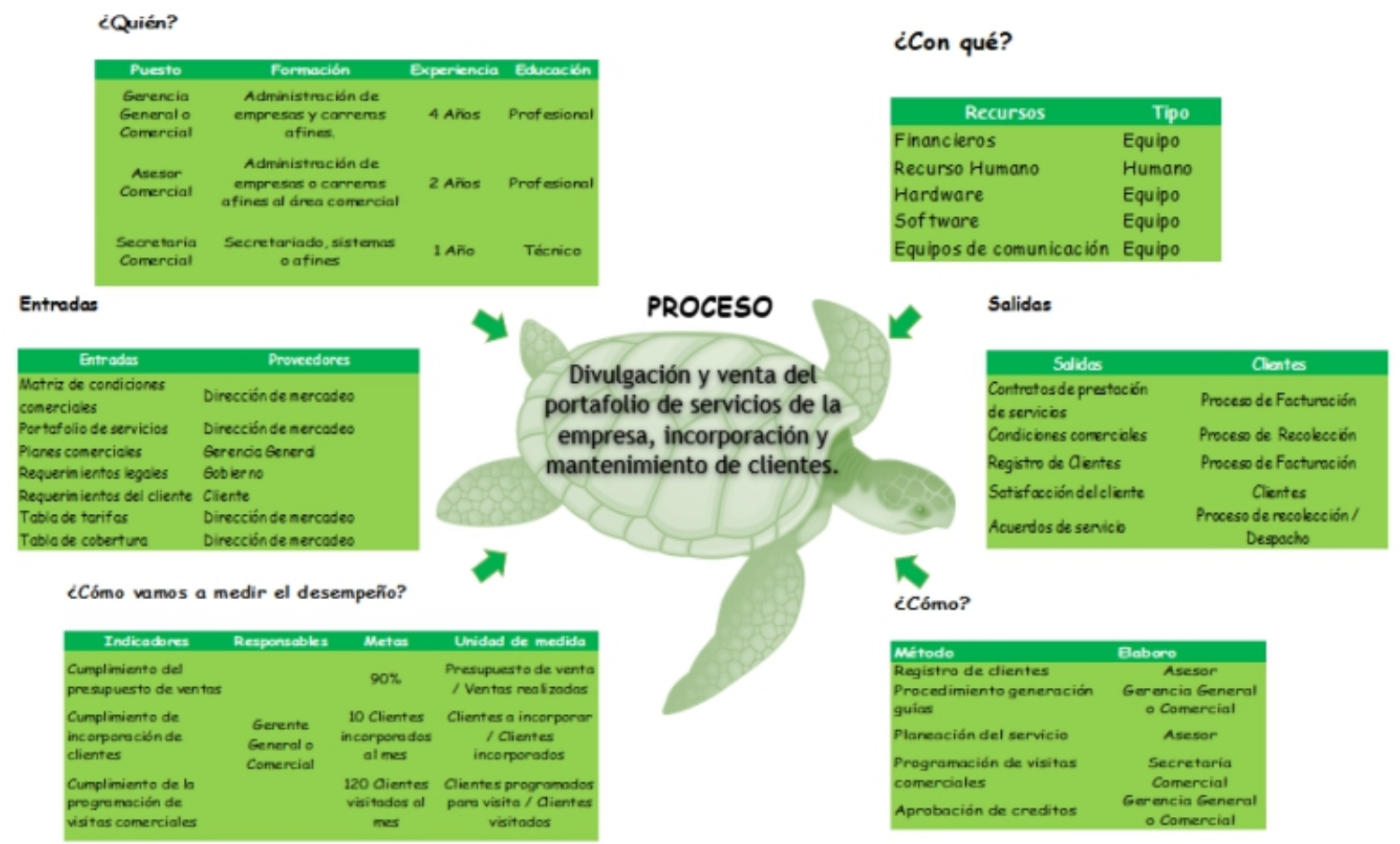

Fig. 9. Diagrama de tortuga para representar la caracterización del nuevo proceso comercial de la empresa 
Los competidores fueron evaluados mediante la herramienta de benchmarking competitiva recolectando información de primera mano.

El proceso comercial fue rediseñado mediante la herramienta QFD, caracterizado y plasmado en un diagrama de tortuga teniendo en cuenta los requerimientos y expectativas del cliente.

Las actividades de seguimiento y control previenen los malos resultados y las desviaciones para el adecuado desarrollo de las actividades del proceso y una buena prestación del servicio.

Como herramientas aplicadas para la mejora continua para el diseño de servicios, la casa de la calidad permite considerar la voz del cliente como parámetros para la determinación de actividades que permitirán satisfacer las necesidades y expectativas, desde una visión holística, ya que no solo mira el cliente, sino que compara las mejoras practicas aplicadas en el mercado y las capacidades que tiene la organización para cumplir estos requerimientos, sobre un análisis cuantitativo que permite calcular la importancia relativa y la importancia absoluta, para determinar el grado de dificultad que representaría la adopción de cada una de las medidas tomadas en el diseño, sin llegar a la subjetividad.

Los parámetros establecidos por el cliente como requerimientos, también son objeto de evaluación, correlacionando con los métodos utilizados por la empresa, permitiendo determinar la fuerza de esta relación, excluyendo aquellas en las cuales la correlación es negativa y extremando esfuerzos sobre actividades que muestran una fuerte relación, dando a entender que las capacidades de la empresa están dispuestas a cumplir con los requerimientos de los clientes, y que los requerimientos entre si se relacionan y que se requiere de unos para soportar los otros, estas relaciones de inclusión permiten extremar los esfuerzos dado que la entrada de una actividad de proceso apalancaría el siguiente proceso.

Trabajo Futuro Con el fin de valorar el impacto del diseño propuesto en la empresa, se propone como investigaciones que se pueden desarrollar en el futuro la evaluación de las mejoras en el diseño del proceso comercial, visto desde la satisfacción de las necesidades y expectativas de los clientes.

\section{Referencias}

Aguilar-Barojas, S. (2005). Fórmulas para el cálculo de la muestra en investigaciones de salud. Salud en Tabasco, 333-338.

Betancourt, D. (5 de Julio de 2016). Ingenio Empresa. Obtenido de www.ingenioempresa.com/arbol-deproblemas.

Bolar, A. A., Tesfamariam, S., \& Sadiq, R. (2017). Framework for prioritizing infrastructure user expectations using Quality Function Deployment (QFD). International Journal of Sustainable Built Environment, 6(1), 16-29. https://doi.org/10.1016/j.ijsbe.2017.02.00

Buonocore, D. (1980). Diccionario de bibliotecología. Buenos aires: Marymar.

Cuatrecasas, L. (2012). Gestión de la Calidad Total. Madrid: Ediciones Díaz de Santos.

Delgado, M., Parra, W., \& Suarez, A. (2015). Diseño de un modelo de mejoramiento continuo en la prestación del servicio aplicado a la cooperativa de educadores Canapro O.C. Bogotá.

Gehisy. (13 de Febrero de 2017). Aprendiendo calidad y adr. Obtenido de https://aprendiendocalidadyadr.com/mapeo-deprocesos-iso-90012015/

Gonzáles, C., Domingo, R., \& Sebastián, M. (2013). Técnicas de mejora de la calidad. Madrid: UNED.

Gutierrez, H. D. (2013). Control Estadístico de la calidad y sesis sigma. Mexico: Mc Graw Hill Education.

Gutierrez, H., Gutierrez, P., Garibay, C., \& Díaz, L. (2014). Análisis multivariado y QFD como herramientas para escuchar la voz del cliente y mejorar la calidad del servicio. Ingeniare, 62-73.

Herrera De la Barrera, J., \& Narváez, C. (2017). Metodología para la comprensión de la voz del cliente en entornos dinámicos utilizando el despliegue de funciones de calidad (QFD). Teknos Revista Científica, 63-72.

Imaroh, T. (2018). Direct Selling Program Strategy Through OnLine Reporting System Design With "IPA \& QFD" Method: (A CASE STUDY AT PT GLOBAL GREEN TRADING). International Journal of Organizational Innovation, 335-344.

INTERNATIONAL STANDARD ORGANITATION ISO. General principles and perspectives of QFD. , $2015 \S$ (2015) 

empresa del sector de transporte de mercancía. Teknos Revista Cientifica, 19(2), 61-74.

Kurtulmuşoğlu, F., \& Pakdil, F. (2017). Combined analysis of service expectations and perceptions in lodging industry through quality function deployment. Total Quality Management \& Business Excellence, 1393-1413.

Llontop, J. (2011). Mejora en el proceso de desarrollo de nuevas líneas de productos basado en la metodología QFD asegurando la calidad del producto a lo largo de su ciclo de vida. Lima.

Lopéz, P. (2016). Herramientas para la mejora de la calidad: métodos para la mejora continua y la solución de problemas. Madrid: FC Editorial.

Madrigal, R. (2018). Control estadístico de la calidad. Ciudad de México: Grupo Editorial Patria.

Medina, A. (2005). Gestión por procesos y creación de valor público: un enfoque analítico. Santo Domingo: INTEC.

Ministerio de la Protección Social. (23 de 04 de 2019). Guía de instrucción Despliegue de la función calidad (QFD). Bogotá, Colombia.

Montgomery, D. (2013). Introduction to Statistical quality control (seventh; John Wiley and sons, Ed.). United States of America

Nuñez, L., Vélez, M., \& Berdugo, C. (2004). Aplicación de una Metodología de Mejora de Procesos basda en el Enfoque de Gestión por procesos, en los Modelos de Excelencia y el QFD en una empresa del sector de confecciones de Barranquilla (Colombia). Ingeniería y Desarrollo, 45-58.

Olaya, E., Cortés, C., \& Duarte, G. (2005). Despliegue de la función calidad (QFD): beneficios y limitaciones detectados en su aplicación al diseño de prótesis mioeléctrica de mano. Ingeniería e Investigación, 3038.

Plazas, F. (2011). Investigación de mercados: un enfoque gerencial. Ediciones de la U.

Miranda, F., Chamorro, A., \& Rubio, S. (2007). Introducción a la gestión de la calidad. Madrid: Delta Publicaciones.

Montesinos, S., Echeverría, Y., Alvarado, A., \& Manzano, M. (2016). Aplicación del QFD y AHP en el diseño de una montura mecánica para manipular una lente sintonizable sólida elástica por compresión. Revista de Ingeniería Industrial, 22-29.

Ruiz, R. (8 de Mayo de 2011). El blog de Ricardo Ruiz de Adana Pérez. Obtenido de http://ricardoruizdeadana.blogspot.com/

Vélez, S. (2011). Herramienta para control y programación de la producción en el sector agrícola. Bogotá.

Yacuzzi, E., \& Martín, F. (2013). QFD: Conceptos, apliacciones y nuevos desarrollos. Argentina.

York, J. (1994). Calitividad: la mejora simultánea de la calidad y la productividad. Barcelona: Marcombo.
Zaïdi, A. (1993). QFD: Despliegue de la función de la calidad. Madrid: Ediciones Diaz de Santos SA. 